\title{
Almacenamiento de carbono en plantaciones de Juglans neotropica Diels, con y sin asocio de Coffea arabica $\mathrm{L}$.
}

\section{Carbon stock in plantations of Juglans neotropica Diels, with and without associate of Coffea arabica L.}

\author{
Jorge Luis Ramírez López ${ }^{1}$, Edwin Andrés Oyos Proaño², Eduardo Jaime Chagna Avila \\ ${ }^{1}$ Universidad Técnica del Norte. Carrera de Ingeniería Forestal, Av. 17 de Julio, 5-21, y José María Córdova, \\ código postal 100105, Ibarra, Ecuador. 0984491594. \\ ${ }^{2}$ Universidad Técnica del Norte. Carrera de Ingeniería Forestal, Av. 17 de Julio, 5-21, y José María Córdova, \\ código postal 100105, Ibarra, Ecuador. \\ ${ }^{3}$ Universidad Técnica del Norte. Carrera de Ingeniería Forestal, Av. 17 de Julio, 5-21, y José María Córdova, \\ código postal 100105, Ibarra, Ecuador. \\ *Correspondencia: jlramirez@utn.edu.ec; eaoyosp@utn.edu.ec; ejchagna@utn.edu.ec
}

Rec.: 10.05.2019. Acept.: 18.10.2019.

Publicado el 31 de diciembre de 2019

\section{Resumen}

$E^{1}$ objetivo de la investigación fue estimar el contenido de carbono almacenado en plantaciones y sistemas agroforestales de Juglans neotropica de la sierra norte ecuatoriana, como un aporte a las estrategias de adaptación y mitigación del cambio climático. Se estimó el carbono de dos plantaciones de 20 años de Juglans neotropica, una pura y otra en asocio con Coffea arabica. La biomasa de árboles y detritus se determinó mediante la relación entre materia fresca y seca. Con un factor de 0.5 se transformó la biomasa en carbono y se realizó fórmulas alométricas para determinar el carbono total de la plantación. El carbono de detritus sobre el suelo se determinó en laboratorio y se extrapoló a unidad de superficie. El carbono almacenado por la plantación pura fue de $13.17 \mathrm{Mg} \mathrm{ha}^{-1}$, mientras que con asocio fue de $16.11 \mathrm{Mg} \mathrm{ha}^{-1}$. Se registraron diferencias en el contenido de carbono de todos los componentes, siendo el fuste el componente que más carbono almacena. Los detritus fue el componente que mostró mayores diferencias entre los sistemas. Se concluye que la plantación en asocio almacenó mayor cantidad de carbono, lo que muestra que la inclusión del café favorece el crecimiento y la producción de biomasa de $J$. neotropica. El fuste fue el componente de mayor almacenaje, hecho que contribuyó a la relación lineal con la regresora $\mathrm{DAP}^{2} \mathrm{Ht}$, obteniéndose ecuaciones con más de un $90 \%$ de ajuste que permiten estimar el carbono almacenado en condiciones silviculturales y edafoclimáticas similares a las del estudio.

Palabras clave: nogal, ecuaciones alométricas, plantaciones, sistemas agroforestales.
$\mathrm{T}$ The objective of the research was to estimate the carbon stock in plantations and agroforestry systems of neotropic Juglans neotropica in the northern Ecuadorian highlands, as a contribution to climate change adaptation and mitigation strategies. Carbon was estimated from two 20-year-old plantations of Juglans neotropica, one pure and one in association with Coffea arabica. The biomass of trees and detritus is determined by the relationship between fresh and dry matter. With a factor of 0.5 the biomass is transformed into carbon and allometric formulas were made to determine the total carbon of the plantation. Detritus carbon was determined in the laboratory and extrapolated to the surface unit. The carbon stock by the pure plantation was $13.17 \mathrm{Mg} \mathrm{ha}^{-1}$, while in association it was $16.11 \mathrm{Mg}$ $\mathrm{ha}^{-1}$. Differences in the carbon content of all components were observed, with the bole being the component that stock the most carbon. Detritus were the component that had the greatest differences between the systems. It is concluded that the plantation in association stock greater amount of carbon, which shows that the inclusion of coffee favors the growth and production of biomass of $J$. neotropica. The bole was the component of greater storage, fact that contributed to the linear relationship with the DAP ${ }^{2} \mathrm{Ht}$ regressor, obtaining equations with more than a $90 \%$ adjustment that allows estimating the carbon stored in silvicultural and edaphoclimatic conditions similar to those of the study.

Keywords: walnut, allometric equations, plantations, agroforestry systems. 


\section{Introducción}

$\mathrm{L}$ a investigación y desarrollo de estrategias de adaptación y mitigación al cambio climático son temas recurrentes en distintos foros y eventos a nivel mundial (IPCC, 2014). Los árboles almacenan grandes cantidades de carbono en su biomasa por medio de la fotosíntesis y en el suelo por la acumulación y descomposición de materia orgánica (Ávila et al., 2001). En este contexto, los bosques y plantaciones son fundamentales en el equilibrio ambiental y el balance de carbono planetario (Yong y Zengyuan, 2015). Adicionalmente a los servicios ambientales de los ecosistemas forestales, sus propietarios pueden obtener ingresos económicos por medio del programa de Reducción de Emisiones por Deforestación y Degradación (REDD+) (Stern y Stern, 2007).

Pese a que en gran medida la función de almacenaje de carbono se atribuye a los bosques nativos, las plantaciones forestales son medios eficaces para su secuestro. Al aprovechar las forestaciones el carbono secuestrado queda inmovilizado en productos elaborados a partir de la madera (López et al., 2018). $\mathrm{El}$ asocio de especies forestales con diferentes cultivos tiene varios beneficios económicos y ambientales (Alonso, 2011). Además de almacenar carbono, el componente forestal de sistemas en asocio contribuye a la recuperación de suelos e incrementa la disponibilidad del recurso hídrico (Ramachandran Nair et al., 2009). Lo mencionado muestra la importancia de plantaciones y sistemas agroforestales para la adaptación y mitigación del cambio climático.

En el Ecuador, Junglas neotropica Diels es la única especie registrada del género, distribuyéndose desde los 1600 a los 2700 m.s.n.m (Palacios, 2016). La madera de esta especie es considerada de alto valor comercial por su alta resistencia y fácil grado de trabajabilidad; haciéndola ideal para la ebanistería fina, carpintería interior, decoración $\mathrm{y}$ fabricación de utensilios torneados y chapas decorativas (Nieto y Rodríguez, 2002). En base a su fruto se fabrican dulces llamados nogadas, patrimonio gastronómico de la provincia de Imbabura (Unigarro, 2010). Los taninos presentes en su corteza y frutos son usados para curtir pieles y en la industria textil (Nieto y Rodríguez, 2002). Las características mencionadas, convirtieron a la especie en una de las principales materias primas de varias actividades económicas de la provincia de Imbabura. Pese a su importancia, esta especie es escasa por su sobreexplotación, encontrándose mayoritariamente en plantaciones y sistemas agroforestales (Kvist et al., 2006). Por lo mencionado es necesaria la generación de datos relacionados a servicios ecosistémicos de la especie, siendo almacenaje de carbono uno de los principales aportes de los ecosistemas forestales a la mitigación del cambio climático.

En este contexto el objetivo de la presente investigación fue estimar el contenido de carbono secuestrado en plantaciones y sistemas agroforestales de Juglans neotropica de la sierra norte ecuatoriana, como un aporte a las estrategias de adaptación y mitigación del cambio climático.

\section{Materiales y métodos}

\section{Área de estudio}

El trabajo de campo se realizó en dos plantaciones de 20 años de Juglans neotropica una pura y otra en asocio con Coffea arabica. Las plantaciones están ubicadas en un mismo predio a $0^{\circ} 23^{\prime} 8.86^{\prime \prime}$ de latitud N y $77^{\circ} 55^{\prime}$ 49.14" de longitud W, en la parroquia de Pimampiro, cantón San Pedro de Pimampiro, provincia de Imbabura. La altitud del ensayo es de 2100 m.s.n.m, con una temperatura promedio de $16^{\circ} \mathrm{C}$ y una precipitación $750 \mathrm{~mm}$ al año (Instituto Nacional de Meteorología e Hidrología [INAMHI], 2017). La superficie de la plantación pura es de $2756.5 \mathrm{~m}^{2}$, mientras que la de en asocio es $1615 \mathrm{~m}^{2}$. Las plantaciones fueron establecidas en las mismas condiciones topográficas y edáficas, con un distanciamiento de $3 \times 3 \mathrm{~m}$. Pese a no recibir tratamientos silviculturales, las plantaciones se consideraron como sanas según el criterio de Murillo \& Camacho (1997).

\section{Cuantificación biomasa individuos muestreados}

Para la cuantificación de la biomasa se realizó el censo total de cada una de las plantaciones, midiéndose las variables dasométricas, diámetro a $1.30 \mathrm{~m}$ (DAP) y altura total $(\mathrm{Ht})$. Se calcularon los estimadores estadísticos: media aritmética $(\mu)$, desviación estándar $(\sigma)$ y coeficiente de variación $(\mathrm{CV})$, de las variables antes mencionadas con el software Infostat versión 2018e. Posteriormente se determinó el número de árboles tipo que servirán de base para la construcción de las ecuaciones alométricas; para dicho efecto se usó el criterio de Picard et al., (2012), el cual determina el tamaño de la muestra en función al número de especies y la homogeneidad del DAP y la Ht de los individuos. Al tratarse de plantaciones homogéneas los individuos muestreados fueron nueve, seleccionados por la cercanía de sus variables dasométricas a la media de cada plantación.

De los árboles tipo se midió su altura con la ayuda de una regla graduada con una presión de 0.1 $\mathrm{m}$; adicionalmente su diámetro mayor y menor con cinta diamétrica y calibrador respectivamente. Con la información levantada se determinó el volumen del fuste con una adaptación de fórmula de Huber, expresada en la ecuación 1. 
$\mathrm{V}=\pi / 4 *[(\mathrm{DM}+\mathrm{dm}) / 2]^{2} * \mathrm{Ht}$

Donde:

$\mathrm{V}=$ Volumen del fuste $\left(\mathrm{m}^{3}\right)$.

$\mathrm{Dm}=$ Diámetro mayor $(\mathrm{m})$.

$\mathrm{dm}$ : Diámetro menor $(\mathrm{m})$.

$\mathrm{Ht}=$ Altura total $(\mathrm{m})$.

El estudio usó un método no destructivo, por lo que se tomó muestras por duplicado del fuste de cada individuo a $1.30 \mathrm{~m}$. Este proceso se realizó con un barreno de Pressler de diámetro $5.15 \mathrm{~mm}$, obteniéndose muestras promedio de $0.05 \mathrm{~m}$ de largo. Las muestras fueron embaladas herméticamente, codificadas y enviadas a laboratorio, en donde fueron pesadas con una balanza de precisión $0.0001 \mathrm{~g}$, registrándose su peso en verde. Posteriormente las muestras fueron secadas al horno a una temperatura de $105{ }^{\circ} \mathrm{C}$ hasta alcanzar su peso contante, registrado como materia seca. La biomasa de las muestras se obtuvo mediante la ecuación 2 (Segura y Andrade, 2008) y fue extrapolada al fuste mediante la relación entre sus volúmenes.

$$
\mathrm{B}=(\mathrm{Pf} * \mathrm{Ms}) / 100
$$

Donde:

$\mathrm{B}=$ biomasa $(\mathrm{kg})$.

$\mathrm{Pf}=$ Peso fresco $(\mathrm{kg})$.

Ms $=$ Materia seca (\%).

Para determinar la biomasa de ramas y hojas se seleccionó una rama bifurcaba directamente del fuste y ubicada en el centro de la copa de cada árbol (valor promedio). La rama fue seccionada y se determinó biomasa usando la misma metodología empleada en las muestras del fuste. La operación se repitió con las hojas de la rama. La biomasa de ramas y hojas de cada individuo se obtuvo con la multiplicación del valor obtenido de la rama de muestra y el total de ramas del árbol.

\section{Cuantificación biomasa componente forestal}

Con los valores de biomasa aérea de los nueve individuos se realizó análisis de regresión múltiple, a fin de determinar la mejor ecuación predictora para cada una de las plantaciones. Las variables regresoras en el análisis múltiple fueron el DAP y la Ht y sus combinatorias. Los supuestos de normalidad, independencia de residuos y homocedasticidad se probaron por medio de las pruebas de Shapiro-Wilk, rachas y análisis gráfico de los residuales del modelo, respectivamente. Para la selección del modelo de mejor ajuste se realizó una depuración por el método "Stepwise", adicionalmente se revisó el coeficiente de Mallows, el error estándar de la estimación, el $\mathrm{R}^{2}$ ajustado y el Criterio de Akaike, para garantizar la selección del modelo de mejor ajuste (Picard et al., 2012). Finalmente, la biomasa total del componente forestal se estimó con la ecuación alométrica de mejor ajuste y los valores de DAP y Ht obtenidos en el censo forestal.

\section{Cuantificación de biomasa de detritus}

Para determinar la biomasa de detritus se establecieron diez parcelas de $0.50 \times 0.50 \mathrm{~m}$ de forma aleatoria en cada una de las plantaciones, el número de parcelas se determinó en función a la metodología usada por (Gutiérrez y Lopera, 2001). En cada unidad de muestreo se tomó el material en descomposición sobre el suelo. Estas muestras fueron codificadas y pesadas en campo, luego se enviaron a laboratorio en donde se siguió el mismo procedimiento establecido para el resto de componentes. El total de biomasa de detritus se obtuvo con la extrapolación del promedio de las unidades de muestreo al total de la superficie de los sistemas estudiados. La biomasa total del componente forestal se estimó con la ecuación alométrica de mejor ajuste y los valores de DAP y Ht obtenidos en el censo forestal.

\section{Cuantificación del carbono}

La cuantificación del carbono aéreo se obtuvo mediante la multiplicación de los valores de biomasa del componente forestal y de detritus por un factor de conversión de 0.5 (Penman et al., 2003).

\section{Resultados y discusión}

\section{Censo forestal}

La plantación con asocio contó con 271 individuos, mientras que la plantación sin asocio con 145. El resumen de los valores de las variables dasométricas y sus estimadores estadísticos se muestran en el Cuadro 1.

El promedio de DAP y $\mathrm{Ht}$ en las dos plantaciones están muy por debajo de los máximos registrados para esta especie. En condiciones óptimas la especie pude alcanzar los $25 \mathrm{~m}$ de altura y $0.4 \mathrm{~m}$ de DAP (Nieto y Rodríguez, 2002). La precipitación registrada en la zona de estudió es menor a la requerida por $J$. neotropica (Nieto y Rodríguez, 2002), lo que explicaría el pobre crecimiento de las plantaciones. Pese a estas limitantes se observa un mayor rendimiento de la plantación en asocio con café. La cantidad de nutrientes adicionales (Andriulo y Irizar, 2017) y una mayor cantidad de agua infiltrada (Gómez et al., 2011), producto del aporte adicional de materia orgánica podría explicar esta diferencia. Adicionalmente la sombra del café reduce la evaporación del agua del suelo (Lin, 2010), aumentando su disponibilidad para el componente forestal. Si bien 
existe un requerimiento hídrico por parte del cultivo, la diferencia de profundidad a la que las raíces toman el recurso minimiza su competencia (Cannavo et al., 2011). Pese a que la densidad de plantación está dentro de los rangos recomendados para fines de producción forestal (Barreto et al., 1990), el crecimiento de las plantaciones limita su rendimiento volumétrico.

\section{Ajuste ecuación alométrica}

La ecuación alométrica de mejor ajuste para la estimación de biomasa de $J$. neotropica, tanto en asocio como sin asocio, tuvo como variable regresora la combinatoria $\mathrm{DAP}^{2} \mathrm{Ht}$. El nivel de ajuste fue alto con $\mathrm{R}^{2}$ superiores a 0.99 (Figuras 1 y 2) y un nivel de significancia por encima del $99 \%$. Además de su efectividad, la ecuación proveniente de la combinatoria muestra una menor complejidad que las ecuaciones obtenidas con las variables por separado. Investigaciones similares encontraron también un mejor ajuste con la combinación de las variables (Pacheco et al., 2007; Ribeiro et al., 2015; Zewdie et al., 2009), esto mejora el proceso de estimación y elimina la necesidad de transformación de las variables.

Las Figuras 1 y 2 muestran la linealidad entre las variables, por lo que se deduce que la variable combinatoria $\mathrm{DAP}^{2} \mathrm{Ht}$ es la regresora de mejor ajuste para la estimación de biomasa. Los análisis de regresión con las variables

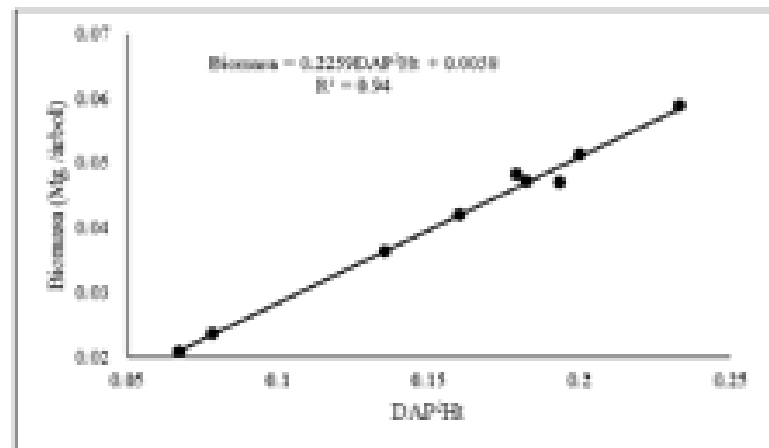

Figura 1. Relación entre biomasa y la variable DA $P^{2} H t$ en plantaciones con asocio

Cuadro 1. Resumen datos dasométricos

\begin{tabular}{|c|c|c|c|c|c|}
\hline Plantación & Estimador & $\begin{array}{l}\text { Ht } \\
(\mathbf{m})\end{array}$ & $\begin{array}{c}\text { DAP } \\
\text { (m) }\end{array}$ & $\begin{array}{c}\mathbf{A B} \\
\left(\mathbf{m}^{2} / \mathbf{h a}\right)\end{array}$ & $\begin{array}{c}V \\
\left(m^{3} / \mathbf{h a}\right)\end{array}$ \\
\hline \multirow{3}{*}{ Sin Asocio } & $\mu$ & 5.77 & 0.12 & 10.91 & 30.97 \\
\hline & $\sigma$ & 1.04 & 0.02 & & \\
\hline & CV (\%) & 18.12 & 19.01 & & \\
\hline \multirow{3}{*}{ Con Asocio } & $\mu$ & 6.59 & 0.13 & 13.01 & 38.40 \\
\hline & $\sigma$ & 1.00 & 0.02 & & \\
\hline & CV (\%) & 15.17 & 18.59 & & \\
\hline
\end{tabular}

Ht: altura total; DAP: diámetro a 1.30 m; AB: área basal; V: volumen; $\mu$ : promedio; $\sigma:$ desviación estándar; CV: coeficiente de variación. 
Almacenamiento de carbono en plantaciones de Juglans neotropica Diels, con y sin asocio de Coffea arabica L.

Cuadro 2. Ecuaciones de mejor ajuste con estadísticos de selección

\begin{tabular}{llllll}
\hline Plantación & \multicolumn{1}{c}{ Ecuación } & $\mathbf{R}^{2}$ aj & EEE & Cp & AIC \\
\hline \multirow{5}{*}{ Con Asocio } & $\mathrm{B}=0.0058+0.2259 \mathrm{Dap}^{2} \mathrm{Ht}$ & 99.5 & 0.0006 & 2.03 & -13.66 \\
& $\mathrm{~B}=-0.0119+0.2976 * \mathrm{Dap}-1.59813 \mathrm{Dap}^{2}+0.2822 \mathrm{Dap}^{2} \mathrm{Ht}$ & 99.7 & 0.0007 & 2.64 & -13.57 \\
& $\mathrm{~B}=-0.00525+0.0021 \mathrm{Ht}+0.2016 \mathrm{Dap}^{2} \mathrm{Ht}$ & 99.6 & 0.0008 & 2.06 & -13.65 \\
& $\mathrm{~B}=0.0093+0.2025 \mathrm{Dap}^{2} \mathrm{Ht}$ & 99.9 & 0.0000 & 1.20 & -14.98 \\
Sin Asocio & $\mathrm{B}=0.0583-0.0093 \mathrm{Ht}-0.4403 \mathrm{Dap}+3.1876 \mathrm{Dap}^{2}+0.0009 \mathrm{Ht}^{2}$ & 99.8 & 0.0004 & 5.00 & -14.78 \\
& $\mathrm{~B}=-0.0033+1.5255 \mathrm{Dap}^{2}+0.0002 \mathrm{Ht}^{2}$ & 99.6 & 0.0007 & 8.28 & -14.38 \\
\hline
\end{tabular}

B: biomasa; $R^{2}$ aj: Coeficiente de determinación ajustado; EEE: Error estándar de la estimación; Cp: Coeficiente de Mallows; AIC: Criterio de Akaike

\section{Cuantificación biomasa}

El total de biomasa aérea de la plantación con asocio fue de $31.63 \mathrm{Mg} \mathrm{ha}^{-1}$, mientras que la biomasa de la plantación sin asocio fue de $26.25 \mathrm{Mg} \mathrm{ha}^{-1}$. Son escasos los estudios desarrollados sobre producción primaria de forestaciones de $J$. neotropica. Las investigaciones sobre la especie se centran en su propagación (Carvajal y Cardona, 1998; López y Piedrahita, 1999; Quintero y Jaramillo, 2012; Vanegas y Rojas, 2018) y en las propiedades medicinales y nutricionales de sus frutos (Juro et al., 2010; Vilcacundo et al., 2018; Vilcacundo et al., 2019). La biomasa promedio por individuo en la plantación con asocio fue de $53.02 \mathrm{~kg}$, mientras sin asocio se registró un valor igual a $34.58 \mathrm{~kg}$. Estos valores están muy por debajo de los $286.80 \mathrm{~kg}$ de Acacia melanoxylon y a los $71.10 \mathrm{~kg}$ de Alnus acuminata, especies nativas usadas en sistemas agroforestales de la sierra ecuatoriana (Riofrío y Grijalva, 2013).

Para las dos plantaciones la mayor cantidad de biomasa se concentró en el fuste. En el caso de la plantación sin asocio, el porcentaje de biomasa concentrado en ramas, hojas y detritus fue superior al de la plantación con asocio, tal como se muestra en la Figura 3.

El porcentaje de biomasa en ramas y hojas es superior a la descrita en estudios de plantaciones forestales con fines madereros (Paixão et al., 2006; Zewdie et al., 2009; Ribeiro et al., 2015). El porcentaje de biomasa correspondiente a ramas y hojas se debe a las características propias de la especie y a la falta de manejo silvicultural de las plantaciones, que permitió

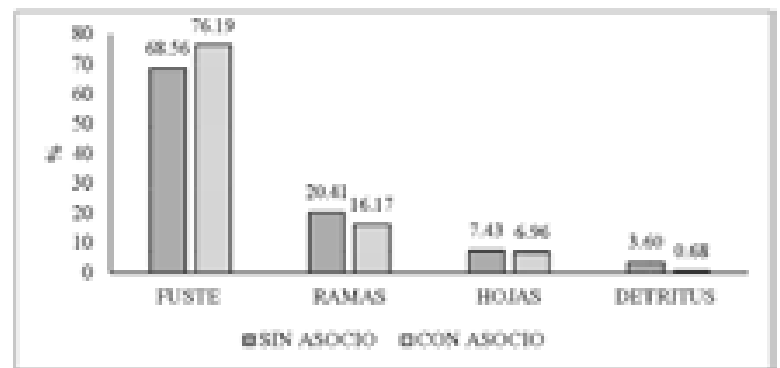

Figura 3. Porcentaje de biomasa por componente el desarrollo de ramas desde alturas más bajas y un mayor volumen de copa (Toro, 2004). La distribución por componente de las plantaciones se asemeja a los descritos en sistemas agroforestales por Tumwebaze et al., (2013), en especial con la plantación en asocio donde la cantidad de detritus es superior debido al aporte de materia orgánica proveniente del cultivo.

\section{Cuantificación carbono}

El contenido de carbono secuestrado en el componente forestal de las dos plantaciones estudiadas (Cuadro 3), es inferior a los $19.99 \mathrm{Mg} \mathrm{ha}^{-1}$ registrado en un sistema agroforestal en asocio con granadilla en una zona cercana a la del presente estudio (Añazco et al., 2019), esto pese a que las plantaciones estudiadas tenían una densidad de individuos ocho veces superior. Lo mencionado confirma que las condiciones edafoclimáticas no fueron favorables para el desarrollo de las plantaciones. Una situación similar se observa si se compara el carbono secuestrado con los $26.64 \mathrm{Mg} \mathrm{ha}^{-1}$ registrados por Zhang et al., (2017) en una plantación de Juglands regia de 13 años en el suroeste de China. Esta situación difiere si se compara con los $16 \mathrm{Mg} \mathrm{ha}^{-1}$ secuestrados por árboles de Juglans nigra de 20 años establecidos en sistemas agroforestales de Ontario. Si bien el contenido de carbono por unidad de superficie es similar, al comparar los $23.30 \mathrm{kgC} /$ árbol secuestrado en promedio en las plantaciones de Pimampiro, con los $139 \mathrm{kgC} /$ árbol secuestrados en Ontario, se observa que

Cuadro 3. Resumen carbono secuestrado por las plantaciones

\begin{tabular}{ccc} 
Componente & Con Asocio & Sin Asocio \\
\hline Forestal $\left(\mathrm{Mg} \mathrm{ha}^{-1}\right)$ & 15.53 & 13.08 \\
Detritus $\left(\mathrm{Mg} \mathrm{ha}^{-1}\right)$ & 0.58 & 0.09 \\
Total $\left(\mathrm{Mg} \mathrm{ha}^{-1}\right)$ & 16.11 & 13.17 \\
\hline
\end{tabular}


las plantaciones estudiadas muestran una menor tasa de secuestro de $\mathrm{C}$.

Pese a la similitud entre los caracteres dendrométricos de las especies (Williams, 1990; Nieto y Rodríguez, 2002; Loewe y González, 2017) y a un mayor número de individuos por hectárea de las plantaciones estudiadas, las diferencias registradas con los otros estudios es considerable. Esta diferencia puede explicarse por el limitado crecimiento de la plantación producto del déficit hídrico.

Sin embargo, el contenido de carbono almacenado por las plantaciones estudiadas es mayor a sistemas agroforestales con especies nativas establecidos en el norte del Ecuador, como por ejemplo los $8.76 \mathrm{Mg} \mathrm{ha}^{-1}$ registrados en un sistema agrosilvícola de Inga insignis en asocio con Phaseolus vulgarisde, los $3.47 \mathrm{Mg} \mathrm{ha}^{-1}$ de Persea americana en asocio con Zea mays o los $0.79 \mathrm{Mg} \mathrm{ha}^{-1}$ de Polylepis racemosa en asocio con pala natural Stipa ichusecuestrados (Añazco et al., 2019). Si bien el contenido de carbono almacenado es mayor que varios sistemas agroforestales, este valor es bajo en relación a las características dasométricas de la especie. Condiciones edafoclimáticas óptimas para el desarrollo de las especies son fundamentales para la fijación de carbono. En este contexto, el asocio con un cultivo mejora la capacidad de fijar carbono de la especie, tal como muestra en este estudio. Esto se debe al aporte adicional de materia orgánica en el suelo que mejora la disponibilidad de nutrientes y la humedad del suelo. (Matus et al., 2000; Gómez et al., 2011).

\section{Conclusiones}

$\mathrm{E}$ xistió una mayor cantidad de carbono almacenado en la plantación con asocio, lo que muestra que la inclusión del café favorece el crecimiento y la producción de biomasa de $J$. neotropica. El componente que registró mayor cantidad de carbono almacenado fue el fuste. Esto explica el ajuste lineal de las ecuaciones alométricas usando como única regresora la variable combinada $\mathrm{DAP}^{2} \mathrm{Ht}$. Las ecuaciones alométricas mostraron un ajuste de más del $90 \%$, volviéndolas herramientas eficaces para la estimación del carbono almacenado en plantaciones de $J$. neotropica en zonas edafoclimáticas similares, con variables dasométricas de fácil medición.

\section{Bibliografía}

Alonso, J. 2011. Los sistemas silvopastoriles y su contribución al medio ambiente. Revista Cubana de Ciencia Agrícola 45(2): 107-115.

Andriulo, A. y Irizar, A. 2017. La materia orgánica como indicador base de calidad del suelo.En: Wilson, M. (Ed.).
Manual de indicadores de calidad del suelo para las ecorregiones de Argentina. Entre Rios, Argentina. p 65.

Añazco, M., Espinosa, A., Chagna, E., Guachamin, V., Ramírez, J., Ocampo, L. y Vizcaíno, M. 2019. Especies forestales y su aporte a la mitigación del cambio climático en los Andes del Ecuador. En: Vera, L.(Ed.). Ciencias ambientales, Base para la sostenibilidad, Memorias del 1er Congreso Internacional de Ciencias Ambientales. Pasto, Colombia. p 59.

Ávila, G., Jiménez, F., Beer, J., Gómez, M. y Ibrahim, M. 2001. Almacenamiento, fijación de carbono y valoración de servicios ambientales en sistemas agroforestales en Costa Rica. Agroforestería En Las Américas 8(30): 32-35.

Avsar, M. 2004. The relationships between diameter at breast height, tree height and crown diameter in Calabrian pines (Pinus brutia Ten.) of Baskonus Mountain, Kahramanmaras, Turkey. Journal of Biological Sciences 4(4): 437-440.

Avsar, M. y Ayyildiz, V. 2005. The relationships between diameter at breast height, tree height and crown diameter in Lebanon cedars (Cedrus libani A. Rich.) of the Yavsan Mountain, Kahramanmaras, Turkey. Pakistan Journal of Biological Sciences 9: 1228-1232.

Barreto, G., Herrera, J. y Trujillo, E. 1990. Juglans neotropica - Plan de acción Forestal para Colombia (Serie N 40). Ministerio de Agricultura- Instituto de Recursos Naturales Renovables y del Ambiente. Bogota, Colombia.

Cannavo, P., Sansoulet, J., Harmand, J., Siles, P., Dreyer, E. y Vaast, P. 2011. Agroforestry associating coffee and Inga densiflora results in complementarity for water uptake and decreases deep drainage in Costa Rica. Agriculture, Ecosystems \& Environment 140(1-2): 1-13.

Carvajal, J. y Cardona, E. 1998. Respuesta de la semilla de cedro negro (Juglans neotropica Diels) a la aplicación de tratamientos pregerminativos. Revista Facultad Nacional de Agronomía Medellín 51(1): 217-235.

Djomo, A., Ibrahima, A., Saborowski, J. y Gravenhorst, G. 2010. Allometric equations for biomass estimations in Cameroon and pan moist tropical equations including biomass data from Africa. Forest Ecology and Management 260(10): 1873-1885.

Gómez, F., Roupsard, O., Maire, G., Taugourdeau, S., Pérez, A., Oijen, M. van, ... Voltz, M. 2011. Modelling the hydrological behaviour of a coffee agroforestry basin in Costa Rica. Hydrology and Earth System Sciences 15(1): 369-392.

Gutiérrez, V. y Lopera, G. 2001. Metodología para la cuantificación de existencias y flujo de carbono en plantaciones forestales. Memorias Simposio Internacional Medición y Monitoreo del la Captura de Carbono en Ecosistemas Forestales: 12.

Instituto Nacional de Meteorología e Hidrología [INAMHI]. 2017. Anuario Meteorológico $\mathrm{N}^{\circ}$ 53-2013. (José 
Olmedo, Ed.). Instituto Nacional de Meteorología e Hidrología, Quito, Ecuador.

IPCC. (2014). Cambio climático 2014: Informe de síntesis. Contribución de los Grupos de trabajo I, II y III al Quinto Informe de Evaluación del Grupo Intergubernamental de Expertos sobre el Cambio Climático. (R.K. Pachauri y L.A. Meyers, Eds.). IPCC, Ginebra, Suiza.

Jenkins, J., Chojnacky, D., Heath, L. y Birdsey, R. 2003. National-scale biomass estimators for United States tree species. Forest Science 49(1): 12-35.

Juro, S., Flores, V., Mendoza, Y. y Del Carpio, C. 2010. Efecto cicatrizante de las diferentes formas farmacéuticas tópicas elaboradas con el extracto hidroalcohólico de Juglans neotropica Diels "nogal" en ratones albinos. Folia Dermatológica Peruana 21(1): 19-24.

Kvist, L., Aguirre, Z. y Sánchez, O. 2006. Bosques montanos bajos occidentales en Ecuador y sus plantas útiles. En: Morales, M., Øllgaard, B., Kvist, L. Borchsenius, F. y Balsle, H. (Eds). Botánica Económica de Los Andes Centrales. La Paz, Bolivia. p 205.

Lin, B. 2010. The role of agroforestry in reducing water loss through soil evaporation and crop transpiration in coffee agroecosystems. Agricultural and Forest Meteorology 150(4): 510-518.

Loewe, M. y González, O. 2017. Nogal Común (Juglans regia): Una Alternativa para Producir Madera de Alto Valor. Ministerio de Agricultura. Santiago, Chile.

López, H., Vaides, E. y Alvarado, A. 2018. Evaluación de carbono fijado en la biomasa aérea de plantaciones de teca en Chahal, Alta Verapaz, Guatemala. Agronomía Costarricense 42(1): 137-153.

López, J. y Piedrahita, E. 1999. Tratamientos pregerminativos aplicados a la semilla de cedro negro (Juglans neotropica) para reducir su periodo de germinación. Memorias Segundo simposio sobre avances en la producción de semillas forestales en América Latina: 18.

Mate, R., Johansson, T. y Sitoe, A. 2014. Biomass equations for tropical forest tree species in Mozambique. Forests 5(3): 535-556.

Matus, F., Maire, G. y Christian, R. 2000. Relación entre la materia orgánica del suelo, textura del suelo y tasas de mineralización de carbono y nitrógeno. Agricultura Técnica 60(2): 112-126.

Murillo, O., y Camacho, P. 1997. Metodología para la evaluación de la calidad de plantaciones forestales recién establecidas. Agronomía Costarricense 21(2): 189-206.

Nieto, V. y Rodríguez, J. 2002. Juglans neotropica Diels. En: Vozzo, J. (Ed.). Tropical Tree Seed Manual. USDA Forest Service. Washington D.C., USA. p 528.

Pacheco, F., Aldrete, A., Gómez, A., Fierros, A., Cetina, V., y Vaquera, H. 2007. Almacenamiento de carbono en la biomasa aérea de una plantación joven de Pinus greggii Engelm. Revista Fitotecnia Mexicana 30(3): 251-254.

Paixão, F., Soares, C., Jacovine, L., Da Silva, M., Leite, H. y
Da Silva, G. 2006. Quantification of carbon stock and economic evaluation of management alternatives in a eucalypt plantation . Revista Arvore 30(3): 411-420.

Palacios, W. 2016. Árboles del Ecuador. Editorial UTN. Ibarra, Ecuador.

Penman, J., Gytarsky, M., Hiraishi, T., Krug, T., Kurger, D., Pipatti, R., ... Tanabe, K.(Eds.). 2003. Good practice guidance for land use, land-use change and forestry. IGES-IPCC, Kanagawa, Japón.

Picard, N., Saint-André, L. y Henry, M. 2012. Manual de construcción de ecuaciones alométricas para estimar el volumen y la biomasa de los árboles: del trabajo de campo a la predicción.FAO. Roma, Italia.

Quintero, O. y Jaramillo, S. 2012. Germination and rescue in vitro of immature embryos of black cedar (Juglans neotropica Diels) . Acta Agronomica 61(1): 52-60.

Ramachandran Nair, P., Mohan Kumar, B. yNair, V. 2009. Agroforestry as a strategy for carbon sequestration. Journal of Plant Nutrition and Soil Science 172(1): 1023.

Ribeiro, S., Soares, C., Fehrmann, L., Jacovine, L. y von Gadow, K. 2015. Aboveground and belowground biomass and carbon estimates for clonal eucalyptus trees in Southeast Brazil . Revista Arvore 39(2): 353-363.

Riofrío, J. y Grijalva, J. 2013. Modelos para estimar la biomasa de especies forestales en sistemas agroforestales de la Ecorregión andina del Ecuador. Memorias VI congreso Forestal Español: 2.

Segura, M. y Andrade, H. 2008. ¿Cómo hacerlo? ¿ Cómo construir modelos alométricos de volumen, biomasa 0 carbono de especies leñosas perennes?. Agroforestería de las Américas, 46: 89-96.

Stern, N., y Stern, N. 2007. The Economics of Climate Change: The Stern Review. Cambridge University Press. Cambridge, Inglaterra.

Toro, J. (2004). Alternativas silvícolas para aumentar la rentabilidad de las plantaciones forestales. Bosque (Valdivia) 25(2): 101-113.

Tumwebaze, S., Bevilacqua, E., Briggs, R. y Volk, T. 2013. Allometric biomass equations for tree species used in agroforestry systems in Uganda. Agroforestry Systems 87(4): 781-795.

Unigarro, C. 2010. Patrimonio cultural alimentario. Ediciones La Tierra, Quito, Ecuador.

Uriel, E. 2013. Introducción a la Econometría. Universidad de Valencia. Valencia, España.

Vanegas, E. y Rojas, I. 2018. Estado del arte, propagación y conservación de Juglans neotropica Diels., en zonas andinas . Madera Bosques, 24(1): e2411560

Vilcacundo, E., Alvarez, M., Silva, M., Carpio, C., Morales, D. y Carrillo, W. 2018. Fatty acids composition of tocte (Juglans neotropica Diels) walnut from Ecuador. Asian Journal of Pharmaceutical and Clinical Research, 11(2): 395-398. 
Vilcacundo, R., Morales, D., León, S., Carpio, C., \& Carrillo, W. (2019). Walnut protein concentrate (Juglans neotropica Diels), gastrointestinal digests and their antioxidant capacity. Italian Journal of Food Science, 31: 93-105.

Wang, J., Zhang, C., Xia, F., Zhao, X., Wu, L. y von Gadow, $\mathrm{K}$ 2011. Biomass structure and allometry of Abies nephrolepis (Maxim) in Northeast China. Silva Fennica, 45(2): 211-226.

Williams, R.1990. Juglans nigra L., black walnut. Silvics of North America 2: 391-399.

Yong, P. y Zengyuan, L. 2015. Forest aboveground carbon mapping using multiple source remote sensing data in the Greater Mekong Subregion. 2015 IEEE International Geoscience and Remote Sensing Symposium (IGARSS): 2035.

Zewdie, M., Olsson, M. y Verwijst, T. 2009. Aboveground biomass production and allometric relations of Eucalyptus globulus Labill. coppice plantations along a chronosequence in the central highlands of Ethiopia. Biomass and Bioenergy 33(3): 421-428.

Zhang, H., Wang, K., Zeng, Z., Du, H. y Zeng, F. 2017. Biomass and carbon sequestration by Juglans regia plantations in the karst regions of southwest China. Forests 8(4): 103.

Zianis, D. y Mencuccini, M. 2003. Aboveground biomass relationships for beech (Fagus moesiaca Cz.) trees in Vermio Mountain, Northern Greece, and generalised equations for Fagus sp. Annals of Forest Science 60(5): 439-448. 